\title{
Difference of Diagnostic Rates and Analytical Methods in the Test Positions of Vestibular Evoked Myogenic Potentials
}

\author{
Ji Hyun Kim, $\mathrm{MD}^{1}$, Jeong Mee Park, $\mathrm{MD}, \mathrm{PhD}^{1}$, Sang Yeol Yong, $\mathrm{MD}^{1}$, \\ Jong Heon Kim, $\mathrm{MD}^{1}$, Hee Kim, $\mathrm{MS}^{2}$, Sang-Yoo Park, MD, $\mathrm{PhD}^{3}$
}

\begin{abstract}
${ }^{1}$ Department of Rehabilitation Medicine, Wonju Severance Christian Hospital, Wonju; ${ }^{2}$ Department of Occupational Therapy, Graduate School, Yonsei University; Department of Occupational Therapy, Wonju Medical Center, Wonju; ${ }^{3}$ Department of Otolaryngology-Head and Neck Surgery, Yonsei University Wonju College of Medicine, Wonju, Korea
\end{abstract}

\begin{abstract}
Objective To compare the differences of diagnostic rates, of the two widely used test positions, in measuring vestibular evoked myogenic potentials (VEMP) and selecting the most appropriate analytical method for diagnostic criteria for the patients with vertigo.

Methods Thirty-two patients with vertigo were tested in two comparative testing positions: turning the head to the opposite side of the evaluating side and bowing while in seated position, and bowing while in supine positions. Abnormalities were determined by prolonged latency of p13 or n23, shortening of the interpeak latency, and absence of VEMP formation.

Results Using the three criteria above for determining abnormalities, both the seated and supine positions showed no significant differences in diagnostic rates, however, the concordance correlation of the two positions was low. When using only the prolonged latency of p13 or n23 in the two positions, diagnostic rates were not significantly different and their concordance correlation was high. On the other hand, using only the shortened interpeak latency in both positions showed no significant difference of diagnostic rates, and the degree of agreement between two positions was low.

Conclusion Bowing while in seated position with the head turned in the opposite direction to the area being evaluated is found to be the best VEMP test position due to the consistent level of sternocleidomastoid muscle tension and the high level of compliance. Also, among other diagnostic analysis methods, using prolonged latency of p13 or n23 as the criterion is found to be the most appropriate method of analysis for the VEMP test.
\end{abstract}

Keywords Vestibular evoked myogenic potentials (VEMP), Patient positioning, Data interpretation

Received July 2, 2013; Accepted October 7, 2013

Corresponding author: Ji Hyun Kim

Department of Rehabilitation Medicine, Wonju Severance Christian Hospital, 20 Ilsan-ro, Wonju 220-701, Korea

Tel: +82-33-741-1455, Fax: +82-33-742-1409, E-mail: trapezium1@hanmail.net

(c) This is an open-access article distributed under the terms of the Creative Commons Attribution Non-Commercial License (http://creativecommons.org/ licenses/by-nc/3.0) which permits unrestricted noncommercial use, distribution, and reproduction in any medium, provided the original work is properly cited. Copyright ( 2014 by Korean Academy of Rehabilitation Medicine 


\section{INTRODUCTION}

Vestibular evoked myogenic potentials (VEMP) is used for diagnosing dizziness in patients. VEMP was first known by Tullio [1] when he noticed that loud sound stimulus can cause dizziness. Bickford et al. [2] confirmed strong sound stimulus results in myogenic potential responses on cervical muscles, and Didier and Cazals [3] assumed this response is derived from the saccule. Many studies following them clarified that the vestibular organ is the origin of this response and named it, VEMP $[4,5]$.

VEMP is generated when the saccule of vestibular system respond to sound stimulus. The restricted biphasic potential with short latency evaluated in the sternocleidomastoid muscle (SCM) is called the VEMP [6]. This reflects the vestibulocollic reflex $[7,8]$ and VEMP is known to be related to the abnormalities of saccule and inferior vestibular nerve [9]. Recent clinical studies support the use of VEMP in diagnosing related diseases, such as the vestibular neuritis, acoustic tumor, and Meniere disease.

Test positions widely used in various laboratories could be classified into two methods. First method is to flex the cervical spine while lying in a supine position. Myogenic potentials could be evaluated while lifting the head up about $30^{\circ}$ in supine, or rotating the lifted head to the opposite from the target side to induce the contraction of ipsilateral SCM. The second method is continuously rotating the head, in sitting position, to the opposite from the testing side or keeping down the tilted head to induce the contraction of ipsilateral SCM [10]. However, standardized testing positions have not yet been established for the patients with dizziness.

Although the analyzing methods of the test results differ depending on the institutions, the general definitions of abnormalities are: 1) absence of VEMP formation when waveforms like p13 and n23 cannot be distinguished with naked eyes and when peak or amplitude cannot be calculated, 2) prolonged latency when the latent periods of each p13 or n23 are 2 standard deviations more than that of the normal population, 3 ) shortened interpeak latency (IPL) when the IPL is 2 standard deviations less than that of the normal population, and 4) VEMP asymmetry is 2 standard deviations more than that of the normal population [11]. VEMP asymmetry refers to the difference between amplitudes of right and left, divided by the sum of amplitudes of right and left (VEMP asymmetry (\%)=(Ar-
$\mathrm{Al} / \mathrm{Ar}+\mathrm{Al}) \times 100 ; \mathrm{Ar}$, amplitude of right; $\mathrm{Al}$, amplitude of left). Since the amplitudes are affected by the threshold, age, testing posture, or muscle tension, this calculation is used to compare the right and left $[11,12]$. However, it is yet to be established which of the various analysis methods above is the most appropriate.

Thus this study has its objective to compare the difference in diagnostic rates of the two widely used testing positions for evaluating VEMP and choosing the one with higher concordance as a diagnosis method.

\section{MATERIALS AND METHODS}

\section{Subjects}

Participants were patients with vertigo, referred from Department of Otolaryngology - Head and Neck Surgery of Wonju Severance Christian Hospital, who were examined for VEMP from May 2011 to August 2011. All the patients participating in this study had symptoms of vertigo with the onsets of within 2 weeks and had no medical or surgical histories. Patients experiencing pain in neck or back during the examinations, or those having general weakness or severe dizziness causing difficulties conducting proper examination, were excluded from the study.

\section{Testing method}

After cleaning the skin with alcohol swabs, $\mathrm{Ag} / \mathrm{AgCl}$ surface electrodes of $19 \mathrm{~mm}$ in diameters were attached. The active electrode was attached to the middle of the SCM of the testing part, the reference electrode was attached to the ipsilateral of lateral sternum's proximal part, and the ground electrode was attached to the front side of the chest. The examinees wore headphones, and the clicking sounds of $95 \mathrm{~dB}$ stimulated their ears of the testing side in $5 \mathrm{~Hz}$, and the ears of the opposite side were shielded with continuous noise of $35 \mathrm{~dB}$. Nicolet Viking IV (Nicolet Biomedical Inc., Madison, WI, USA) was used to measure the myogenic potential, and two hundred responses were averaged. Testing positions were turning and lowering the head to the opposite of the testing side in seated position (position 1), and turning the head to the opposite of the testing side and holding the headup about $10 \mathrm{~cm}$ in supine position (position 2). When testing in the seated position, a patient was seated upright on a stool without the back or the headrest. When testing in the supine po- 

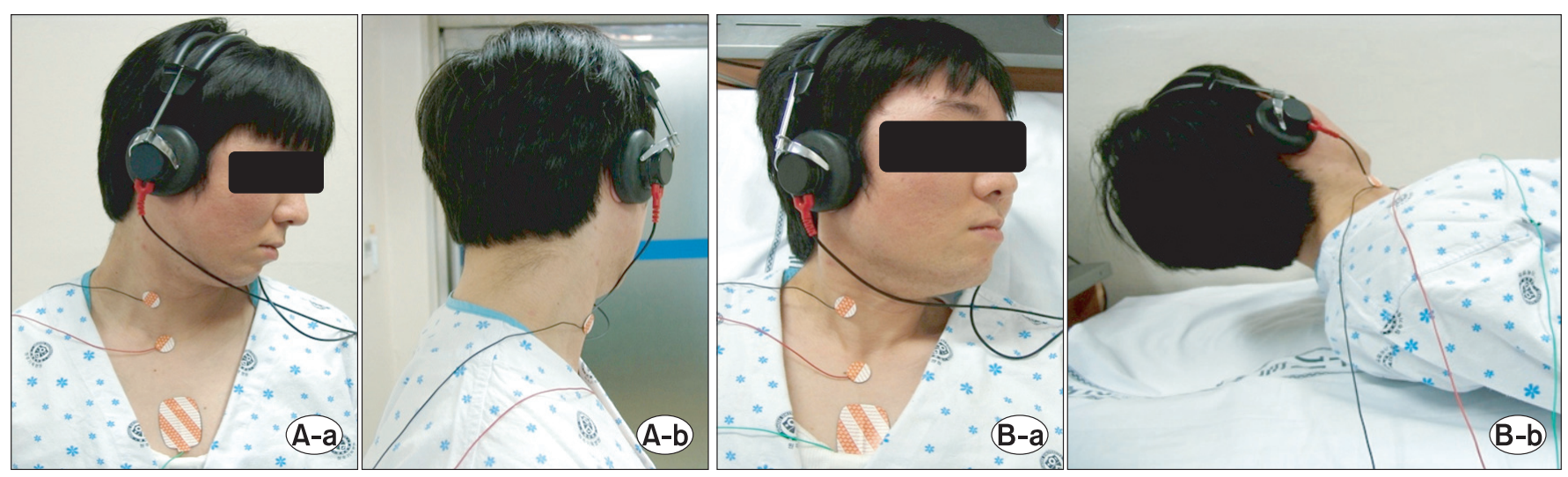

Fig. 1. Testing position. (A) Lowering one's head toward the opposite side of the testing side in seated (position 1: a, anterial; b, lateral). (B) Lifting one's head $10 \mathrm{~cm}$ to the opposite side of the testing side in supine (position 2: a, anterial; b, lateral).

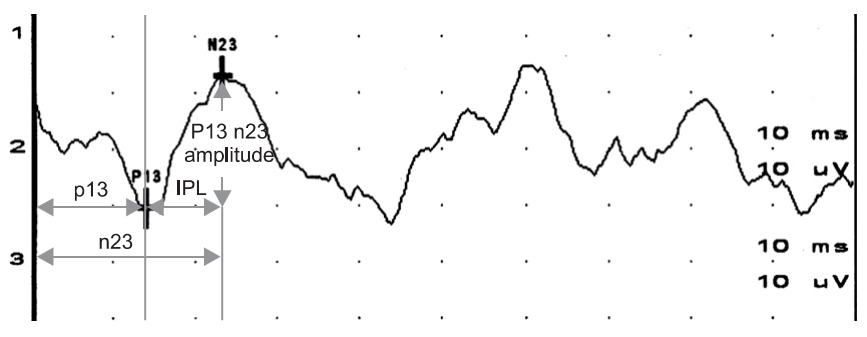

Fig. 2. Typical response and measurement parameters of vestibular evoked myogenic potentials. IPL, interpeak latency.

sition, a patient was laid down straight without a pillow (Fig. 1). Each examinee was first examined in the sitting position followed by the examination in the supine position after ten minutes of resting time. In both the positions, the test was performed on the right-hand side and then on the left-hand side.

\section{Analytical standard}

First positive peak (p13), second negative peak (n23), and amplitudes, which is the myogenic potential difference between the two peaks of both positions, were measured and recorded (Fig. 2).

Using the results above, diagnoses of test parameters were defined as follows: 1) Prolonged latency: latency of p13 or n23 are each prolonged more than two standard deviation ( $\pm 2 \mathrm{SD}$ ) of the normal data; 2) shortened IPL: when the IPL (the difference between the latency of p13 and $\mathrm{n} 23)$ is less than two standard deviation $( \pm 2 \mathrm{SD})$ of the normal data; 3 ) absence of VEMP formation: when waveforms such as p13 or n23 cannot be identified with

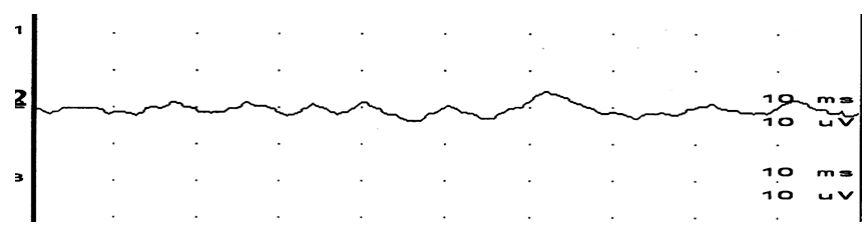

Fig. 3. An example of absence of vestibular evoked myogenic potentials formation.

the naked eyes, and when peaks or amplitudes cannot be measured (Fig. 3).

Abnormality was decided based on the above three criteria and abnormality in each testing position was comparatively analyzed based on each standard. As for the normal value of each indicator, the normal values measured by using clicking sounds in seated posture and reclining posture, as published by Goh [11], were used as references.

\section{Statistical analysis}

Paired t-test was performed to evaluate differences in results according to the test positions. Comparing the abnormality in each testing position as diagnostic analytical method was the McNemar test among cross-tabulations. For the two statistical tests above, any p-value of 0.05 or below was considered as being significant. Kappa number was compared to test the concordance correlation of the two positions when using each analytical method. Kappa number was considered fair when $\kappa \geq 0.4$ and good when $\kappa \geq 0.7$.

All participants were given full information of this study and it was conducted with their voluntary consent. 


\section{RESULTS}

A total of thirty-two patients with vertigo (mean age, $50.8 \pm 14.6$ years) participated in this study; 13 were male patients (mean age, $50.2 \pm 20.3$ years) and 19 were female patients (mean age, 51.3 \pm 9.7 years). Comprehensive test results of the patients participating in this study revealed that 10 patients had benign paroxysmal positional vertigo (BPPV: 4 males and 6 females), 18 had Meniere disease (7 males and 11 females), 1 had vestibular neuritis (1 male and 0 female), and 3 patients were diagnosed with small vessel disease of the cerebrovascular system ( 1 male and 2 females) (Table 1).

For the right-hand side, p13 was measured as $14.51 \pm$ $3.23 \mathrm{~ms}$ in seated position and $13.69 \pm 5.04 \mathrm{~ms}$ in supine position, n23 was $22.61 \pm 3.75 \mathrm{~ms}$ in seated position and $21.98 \pm 6.83 \mathrm{~ms}$ in supine position, and IPL was $8.10 \pm 2.49$ $\mathrm{ms}$ in seated position and $8.29 \pm 3.07 \mathrm{~ms}$ in supine posi-

Table 1. Patient characteristics

\begin{tabular}{lcc}
\hline \multicolumn{1}{c}{ Characteristic } & Male & Female \\
\hline Age (yr) & $50.2 \pm 20.3$ & $51.3 \pm 9.7$ \\
Sex & 13 & 19 \\
Diagnosis & & \\
BPPV & 4 & 6 \\
Meniere disease & 7 & 11 \\
Vestibular neuritis & 1 & 0 \\
\hline $\begin{array}{l}\text { Small vessel disease on } \\
\text { cerebrovascular system }\end{array}$ & 1 & 2 \\
\hline
\end{tabular}

BPPV, benign paroxysmal positional vertigo.

Table 2. Comparison of test parameters between seated position and supine position

\begin{tabular}{crrr}
\hline \multirow{2}{*}{ Test indicator } & \multicolumn{2}{c}{ Latency (ms) } & \multirow{2}{*}{ p-value } \\
\cline { 2 - 3 } & \multicolumn{1}{c}{ Sit } & Supine & \\
\hline Right side & & & \\
p13 & $14.51 \pm 3.23$ & $13.69 \pm 5.04$ & 0.35 \\
n23 & $22.61 \pm 3.75$ & $21.98 \pm 6.83$ & 0.60 \\
IPL & $8.10 \pm 2.49$ & $8.29 \pm 3.07$ & 0.74 \\
Left side & & & \\
p13 & $15.58 \pm 3.96$ & $14.09 \pm 4.99$ & 0.16 \\
n23 & $23.86 \pm 3.94$ & $21.98 \pm 7.35$ & 0.16 \\
IPL & $8.29 \pm 2.23$ & $7.89 \pm 3.33$ & 0.52 \\
\hline
\end{tabular}

Values are presented as mean \pm standard deviation. IPL, interpeak latency. tion. For the left-hand side, p13 was measured 15.58 \pm 3.96 $\mathrm{ms}$ in seated position and $14.09 \pm 4.99 \mathrm{~ms}$ in supine position, $\mathrm{n} 23$ was $23.86 \pm 3.94 \mathrm{~ms}$ in seated position and $21.98 \pm 7.35 \mathrm{~ms}$ in supine position, IPL was $8.29 \pm 2.23 \mathrm{~ms}$ in seated position and $7.89 \pm 3.33 \mathrm{~ms}$ in supine position. All parameter values between the two positions showed no differences ( $p>0.05)$ (Table 2).

When abnormality is defined by any of the three criteria, which are prolonged latency, shortened IPL, and absence of VEMP formation, the diagnostic rate in sitting and supine position were not significantly different in both right ( $\mathrm{p}=0.549)$ and left $(\mathrm{p}=0.549)$ sides. Yet, the concordance correlation of both positions showed low level of 0.185 for right side and 0.054 for left side (Fig. 4).

When abnormality is defined by the prolonged latency among three criteria, diagnostic rate of both postures were not significantly different in both right $(\mathrm{p}=0.453)$ and left $(p=0.453)$ sides and concordance correlation showed high level which were 0.545 for right side and 0.563 for left side (Fig. 5). However, when shortened IPL was the only standard for defining abnormality, although the diagnostic rate of both posture were not significantly different for both right $(\mathrm{p}=0.774)$ and left $(\mathrm{p}=0.804)$ sides, concordance correlation were low which were 0.250 for right side and 0.000 for left side (Fig. 6). Also among the three criteria for the methods of diagnosis, using the absence of VEMP formation did not have enough to find the significance in both positions.

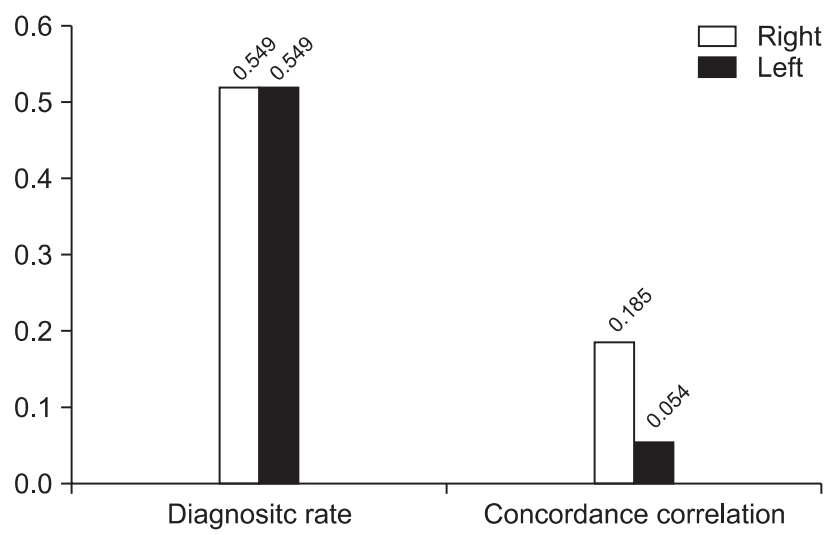

Fig. 4. In the case of defining as a disorder when any of the three diagnosis criteria (prolonged latency, shortened interpeak latency, and absence of vestibular evoked myogenic potentials formation) shows abnormality: diagnosis rates of both positions showed no significant differences in both sides but concordance correlation was low. 


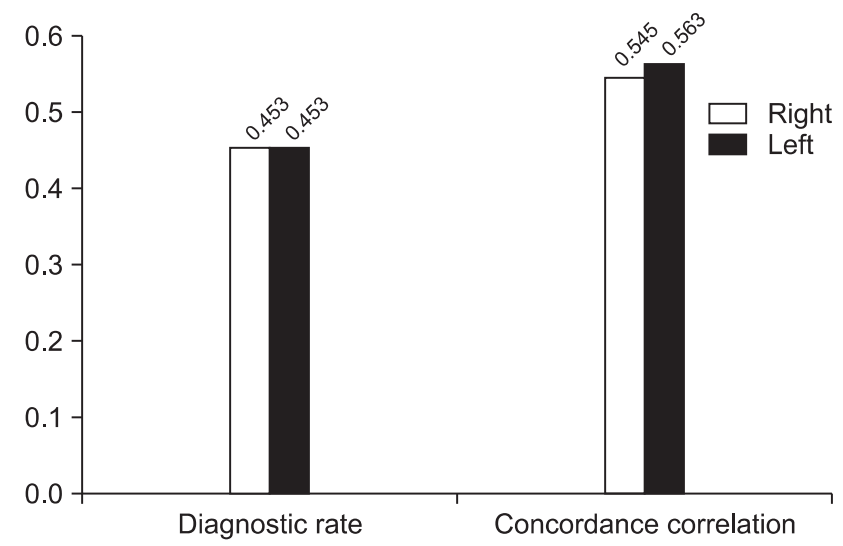

Fig. 5. In the case of defining disorder solely by prolonged latency: diagnosis rates of both positions showed no significant differences in both sides and concordance correlation was high.

\section{DISCUSSION}

VEMP is known to be a reflex that passes the saccule, inferior vestibular nerve, medial vestibular nucleus, and vestibulocollic reflex of the cervical muscle, respectively [6]. Supported by many studies, changes in the results of VEMP are useful for diagnosing many diseases, such as Meniere disease, BPPV, vestibular neuritis, labyrinthitis, and acoustic tumor. Despite its utility of diagnosing diverse diseases, VEMP has a drawback of low reliability in the results due to many variables, such as testing position, muscle tone of SCM, and type and intensity of the stimulation tone affecting the test results. However, the results of recent studies report the pros and cons and the appropriate testing methods to enhance the reliability. The testing postures while examining the VEMP differ for each researcher, and the standard for testing posture and diagnostic analytical method is not yet to be settled. Thus, this study was processed to find the most effective testing position and the appropriate analytical diagnostic method, considering the effects of diverse factors on the results of VEMP test.

Classified as the early and late responses, vestibular system is the origin of the early response and cochlea element is presumed to be the origin of the late response of the VEMP $[7,13]$. Thus, this study only measured the early positive peak (p13) and negative peak (n23), and used the latency of p13 and n23, IPL, and amplitude as a parameter for the test.

As mentioned in the introduction, four analysis meth-

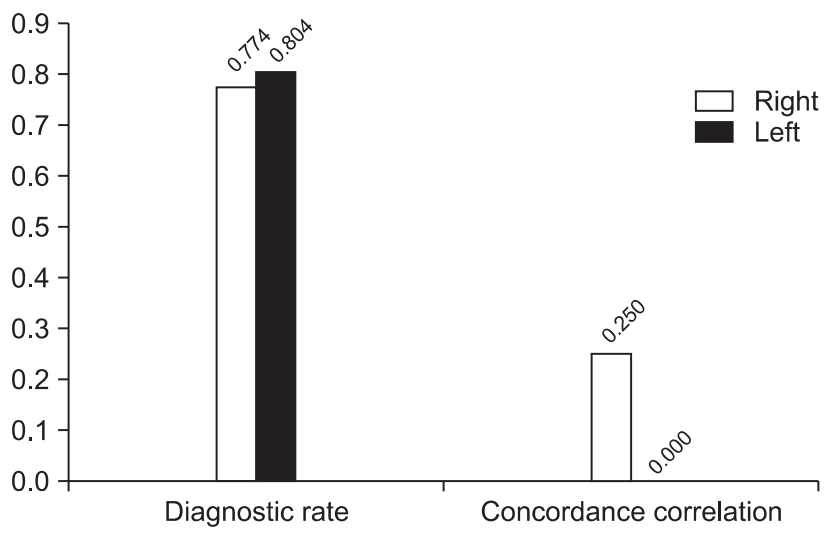

Fig. 6. In the case of defining disorder by shortened interpeak latency alone: diagnosis rates of both positions showed no significant differences in both sides but concordance correlation was low.

ods are generally used in interpretation of the VEMP test. However, since amplitudes of both sides are greatly affected by the patient's systematic conditions, degree of compliance, muscle fatigue, etc., we compared only three of the test methods besides VEMP asymmetry for the appropriateness of analysis methods in this study. Among them, defining abnormalities using only the prolonged latency of p13 or $\mathrm{n} 23$ showed no significant difference in the diagnostic rate of both right and left sides, according to the postures, such as supine and seated. Also, concordance correlation of each posture showed high level. Thus, when diagnosing abnormality solely based on the prolonged latency of p13 or n23, testing posture have less effect on the appropriate diagnosis.

For the case of considering all three evaluation criteria and for the case of only considering decrease of IPL, the low degree of concordance correlation between test positions is thought to be related to compliance for test positions. The test method, of having the patient lie down and raise the head, requires the patient to maintain the position for the entire length of the test. This results in fatigue of the muscles and makes it difficult to maintain a consistent level of muscle tension, with the possibility of compromising on accuracy of the test results $[10,12]$. In fact, we could obtain VEMP waveforms in all the tests we performed in the seated position, but testing in the supine position sometimes induced inconsistent contractions of the SCM muscle with absence of VEMP formation ( 4 cases: 3 persons, 4 tests). Patients in all of these cases complained about the difficulty of maintaining the 
test position, and two of these cases showed normal responses in the seated position. However, there have not been detailed studies concerning correlation of test positions or degree of maintaining contractility of the SCM and the IPL value, and further studies are thought to be necessary on this correlation in the future.

Absence of VEMP formation, which is one of the absolute standards used as judging abnormalities in many studies, was not adequately shown in this study to decide whether or not it is appropriate. However Bickford et al. [2] thought that this reflex is caused by vestibular system rather than the cochlea, from observing patients with total deafness having intact vestibular function response while ones with hearing loss and without vestibular function do not. Case of a patient who lost one's vestibular function by streptomycin infection in one's ear [14], or another case where initial waveform (p13, n23) of VEMP disappeared after amputating one's vestibular nerve, support [5] the fact that VEMP originated from vestibular organ and appears as myogenic potential of cervical muscles. Even though confirming the abnormality using the absence of VEMP formation, regardless of posture, was not available, it is considered that when VEMP was not developed it can be a criterion to judge that vestibular system is abnormal. This result is concurrence to that of Kim et al. [15], whose conclusion included prolonged latency and VEMP asymmetry to the criteria of VEMP, rather than solely using absence of VEMP formation to enhance the diagnostic value.

Common postures for testing VEMP are causing contraction of ipsilateral SCM, by flexing the neck or turning the head in supine or seated positions. Recently, combining both postures to conduct examinations is discussed in the results of the studies [10]. Thus, in order to induce strong contraction of the ipsilateral SCM, flexing and turning the neck to the opposite side of the testing side was used. The measure was conducted not only in the supine position, but also in the sitting position to compare the difference in accessibility of flexing the neck in both positions.

Since each test position had different normal ranges of test parameters; the measurement values were not compared individually, but the ratios of abnormalities were compared between the test positions. There were no significant differences in diagnostic rates between the two postures for both the right side and the left side, under the following three diagnostic analysis methods of the VEMP test: determining abnormality when any of the three criteria (prolonged latency, shortened IPL, and absence of VEMP formation) is shown as abnormal; determining abnormality only based on prolonged latency; and determining abnormality only based on shortened IPL. Thus, VEMP can be valuable in turning the head to the opposite side of the testing one, then dropping one's head in seated position (position 1), turning the head to the opposite side, then raising one' head $10 \mathrm{~cm}$, and in supine position (position 2). Nevertheless, when considering the concordance correlation above, it was found that the error of yielding different evaluation results could be reduced by using prolonged latency as the only criterion.

In addition to the results above, some participants claimed difficulty of maintaining the posture and of the pain in the neck and the back, during progress of the test when testing was done in supine position (position 2). Considering the fact that many of the patients with dizziness are elderlies or patients who have been lying in bed for a long time, the supine position (position 2) can cause more difficulty in maintaining the constant contraction of the SCM compared to the seated position (position 1).

In concurrence to the results of the former studies, processing the test in supine position with the head raised up can cause tension in the SCM and the amplitude can be shown larger [16]. Yet, the difficulty of enduringly the posture during the test can result in irregular results and fatigue in muscles, which can affect the test results of opposite side [17]. On the other hand, the seated posture, with the patient's head turned, has a high rate of compliance for maintaining the test posture $[10,16]$ among the patients suffering from disability of cervical movement, due to disorders of neck muscles and neck bones, and particularly among the children and the elderlies [12].

Previous studies showed low measurement rates and high false negative rates [16], when the test was performed seated with the head turned to the opposite side of the testing one. However, according to Lee et al. [12], all of the fifty-six participants succeeded in recording their measurement, resulting in a $100 \%$ of measurement rate in the study of turning the head in seated position with the normal population.

Although clinical applications of the VEMP test is gradually increasing as a diagnosis tool for assessing the state 
of the inferior vestibular nerve, it is generally thought that VEMP alone is inadequate for confirming the lesion. Considering various measurement-related variables, more future studies are still necessary before clinical applications of the VEMP test are fully established. Also, there need to be more studies concerning diagnostic value, required for more widespread clinical application of the VEMP test, including test positions and adequacy of analysis methods, which were covered in this study. For instance, there have been many studies concerning the application of VEMP in the diagnosis of acoustic neuroma, yielding diagnostic value in a wide range of $66.7 \%$ to $80.8 \%$ [18-21]. However, test methods and analysis methods of diagnosis used in these studies vary greatly, and therefore, direct comparisons of results are rather difficult. Provided that future studies address this issue, the VEMP test would see greater clinical applications with a higher level of reliability.

Limitations of this study were as follows. First of all, evaluation of VEMP asymmetry, which is one of the commonly utilized criteria, was not executed. This was because it is difficult to apply the objective evaluation criteria for the general vertigo patients and the elderly patients, since amplitudes are greatly affected by the patients' systematic conditions, degrees of compliance, muscle fatigues, etc., as mentioned earlier. However, VEMP asymmetry could be a criterion, in the position of turning the head to the opposite side or turning the head to the opposite side and then tilting the head in the seated position; this has comparatively high compliance, and future studies should test the reliability of it.

Secondly, regardless of the position, the frequent absence of VEMP formation results in insufficiency for analyzing the appropriateness of utilizing this for the diagnoses of dizziness in this study. Further studies should gather more participants to analyze it.

Thirdly, considering the reliability, this study suggested that the most appropriate diagnostic evaluation method was to use prolonged latency for determining abnormality of VEMP. However, this excludes diagnostic evaluation methods, considered in many preceding studies so far, with the possibility of increasing the false negative rate. Therefore, it is thought that future studies should include this comparison for greater number of patients.

In conclusion, the authors think that the seated posture with the patient's head turned and bowed in the opposite direction of the area being tested (posture 1) is a good posture for the VEMP test, because it can help to maintain a stable level of tension in the SCM and also can help to yield a high level of compliance. The authors also think that using prolonged latency of p13 or n23 as the criterion, among other diagnostic analysis methods, is the most appropriate method of analysis for the VEMP test.

\section{CONFLICT OF INTEREST}

No potential conflict of interest relevant to this article was reported.

\section{REFERENCES}

1. Tullio P. Das ohr und die entstehung der sprache und schrift. Berlin: Urban \& Schwarzenberg; 1929.

2. Bickford RG, Jacobson JL, Cody DT. Nature of average evoked potentials to sound and other stimuli in man. Ann N Y Acad Sci 1964;112:204-23.

3. Didier A, Cazals Y. Acoustic responses recorded from the saccular bundle on the eighth nerve of the guinea pig. Hear Res 1989;37:123-7.

4. Ferber-Viart C, Dubreuil C, Duclaux R. Vestibular evoked myogenic potentials in humans: a review. Acta Otolaryngol 1999;119:6-15.

5. Colebatch JG, Halmagyi GM. Vestibular evoked potentials in human neck muscles before and after unilateral vestibular deafferentation. Neurology 1992;42:16356.

6. Mudduwa R, Kara N, Whelan D, Banerjee A. Vestibular evoked myogenic potentials: review. J Laryngol Otol 2010;124:1043-50.

7. Colebatch JG, Halmagyi GM, Skuse NF. Myogenic potentials generated by a click-evoked vestibulocollic reflex. J Neurol Neurosurg Psychiatry 1994;57:190-7.

8. Murofushi T, Halmagyi GM, Yavor RA, Colebatch JG. Absent vestibular evoked myogenic potentials in vestibular neurolabyrinthitis: an indicator of inferior vestibular nerve involvement? Arch Otolaryngol Head Neck Surg 1996;122:845-8.

9. Murofushi T, Curthoys IS. Physiological and anatomical study of click-sensitive primary vestibular afferents in the guinea pig. Acta Otolaryngol 1997;117:66-72.

10. Kim JD, Goh EK, Lee YO, Kong SK, Cho KS, Chon KM. The effects of test positions and acoustic stimulations 
on the vestibular evoked myogenic potentials. J Korean Bal Soc 2007;6:21-8.

11. Goh EK. Vestibular evoked myogenic potential. J Clin Otolaryngol Head Neck Surg 2008;19:13-7.

12. Lee SK, Cha CI, Jung TS, Park DC, Yeo SG. Age-related differences in parameters of vestibular evoked myogenic potentials. Acta Otolaryngol 2008;128:66-72.

13. Ferber-Viart C, Duclaux R, Colleaux B, Dubreuil C. Myogenic vestibular-evoked potentials in normal subjects: a comparison between responses obtained from sternomastoid and trapezius muscles. Acta Otolaryngol 1997;117:472-81.

14. Cody DT, Bickford RG. Averaged evoked myogenic responses in normal man. Laryngoscope 1969;79:40016.

15. Kim H, Cha CI, Byun JY, Moon JH, Hong SM, Kim KH. Clinical usefulness of VEMP(vestibular evoked myogenic potential) in the evaluation of dizzy patients. Korean J Otolaryngol-Head Neck Surg 2003;46:391-5.

16. Wang CT, Young YH. Comparison of the head elevation versus rotation methods in eliciting vestibular evoked myogenic potentials. Ear Hear 2006;27:376-81.

17. Park SH, Cha CI, Kim KH, Kim H, Hwang MG, Hong NP. The clinical significance of vestibular evoked myogenic potential evoked by click sound. Korean J Otolaryngol-Head Neck Surg 2001;44:1253-8.

18. Ahn YJ, Hong SK, Kim JS, Koo JW. Diagnostic value of vestibular evoked myogenic potential in acoustic neuroma. J Korean Bal Soc 2007;6:138-42.

19. Matsuzaki M, Murofushi T, Mizuno M. Vestibular evoked myogenic potentials in acoustic tumor patients with normal auditory brainstem responses. Eur Arch Otorhinolaryngol 1999;256:1-4.

20. Murofushi T, Matsuzaki M, Mizuno M. Vestibular evoked myogenic potentials in patients with acoustic neuromas. Arch Otolaryngol Head Neck Surg 1998;124:509-12.

21. Patko T, Vidal PP, Vibert N, Tran Ba Huy P, de Waele C. Vestibular evoked myogenic potentials in patients suffering from an unilateral acoustic neuroma: a study of 170 patients. Clin Neurophysiol 2003;114:1344-50. 